University of Nebraska - Lincoln

DigitalCommons@University of Nebraska - Lincoln

5-3-2007

\title{
Coupled precession modes in indirect exchange-coupled [Pt/ Co]-Co thin films
}

Steven A. Michalski

University of Nebraska-Lincoln, smichalski2@unl.edu

Jian Zhou

University of Nebraska-Lincoln

Ralph Skomski

University of Nebraska-Lincoln, rskomski2@unl.edu

Roger D. Kirby

University of Nebraska-Lincoln, rkirby1@unl.edu

Follow this and additional works at: https://digitalcommons.unl.edu/physicsskomski

Part of the Physics Commons

Michalski, Steven A.; Zhou, Jian; Skomski, Ralph; and Kirby, Roger D., "Coupled precession modes in indirect exchange-coupled [Pt/Co]-Co thin films" (2007). Ralph Skomski Publications. 42.

https://digitalcommons.unl.edu/physicsskomski/42

This Article is brought to you for free and open access by the Research Papers in Physics and Astronomy at DigitalCommons@University of Nebraska - Lincoln. It has been accepted for inclusion in Ralph Skomski Publications by an authorized administrator of DigitalCommons@University of Nebraska - Lincoln. 


\title{
Coupled precession modes in indirect exchange-coupled [Pt/Co]-Co thin films
}

\author{
Steven Michalski, ${ }^{\text {a) }}$ Jian Zhou, ${ }^{\text {b) }}$ Ralph Skomski, and Roger D. Kirby \\ Department of Physics and Astronomy, University of Nebraska, Lincoln, Nebraska 68588 \\ and Nebraska Center for Materials and Nanoscience, University of Nebraska, Lincoln, Nebraska 68588
}

(Presented on 10 January 2007; received 2 November 2006; accepted 20 December 2006; published online 3 May 2007)

\begin{abstract}
Magneto-optical measurements are used to investigate Pt-Co and Co layers exchange-coupled by a Pt layer. The magnetization precession was measured in a pump-probe experiment using a femtosecond laser with direct optical excitation. The competing magnetic anisotropies of the layers yield a noncollinear spin structure with a field-dependent angle between the layers. Two modes, a ferromagnetic or "acoustic" mode and an antiferromagnetic or "optic" mode, are identified from the Fourier-transformed excitation spectra, and the obtained line positions are used to estimate the interlayer exchange constants. () 2007 American Institute of Physics. [DOI: 10.1063/1.2712320]
\end{abstract}

\section{INTRODUCTION}

Exchange-coupled magnetic thin films are scientifically interesting and important in various areas of nanotechnology, such as magnetic recording and field sensors. Traditionally, emphasis has been on thin films with in-plane or perpendicular magnetization, ${ }^{1,2}$ but this paper deals with configurations such as easy-cone and noncollinear magnetism. These spin structures, well known in bulk magnets, ${ }^{3}$ reflect competing interactions, such as competing magnetic anisotropies. We investigate spin reorientations realized by exchange-coupling a layer with perpendicular anisotropy to a layer having the easy axis in the film plane. By varying the thickness of an intervening Pt spacer layer (which determines the interlayer exchange $J$ ) and the perpendicular magnetic anisotropy, we control the magnetic properties, a feature of great practical importance.

Another key aspect of the paper is the used experimental method, namely, a pump-probe technique using femtosecond laser pulses. ${ }^{4-6}$ We will use the method to analyze uniform resonance modes in our films and to estimate $J$. There are two resonance modes in our system that are nearly out of phase with each other. A large part of the paper is actually concerned with evaluation of the pump-probe data by Fourier transformation and micromagnetic modeling. Since the two modes are nearly out of phase, the Fourier transform usually contains only one well defined broad peak and a narrow shoulder, making it difficult to accurately determine the frequencies of the modes

\section{EXPERIMENT}

\section{A. Sample preparation and characterization}

All our samples were produced by magnetron sputtering, sputtered with a base pressure of approximately 3 $\times 10^{-7}$ Torr onto water cooled $\mathrm{Si}(100)$ substrates with a

\footnotetext{
${ }^{a)}$ Electronic mail: michalsk@unlserve.unl.edu

${ }^{b)}$ Present address: Department of Physics, University of California, Berkeley, CA 94720
}

$1.5 \mathrm{~nm}$ Pt seed layer and a $2 \mathrm{~nm}$ Pt protective overcoat. Our samples are $\mathrm{Pt}-\mathrm{Co}$ multilayers with the structure [Pt $0.5 \mathrm{~nm} /$ Co $0.4 \mathrm{~nm}$ ] $\times 4$, which have perpendicular magnetic anisotropy (PMA) exchange coupled to $5 \mathrm{~nm}$ Co layers with in-plane magnetic anisotropy. The exchange is indirect, realized, and tuned by an intervening Pt layer of varying thickness $(0.3-30 \mathrm{~nm})$. Figure 1 shows the basic geometry and indicates the magnetization directions in each layer without interlayer coupling (a) and with ferromagnetic interlayer coupling (b). Samples with Pt spacer layers larger than $1.2 \mathrm{~nm}$ show clear evidence of two mode behavior; thinner layers do not yield a clear signal due to direct coupling. The static magnetic properties were measured by alternating gradient force magnetometer (AGFM), magneto-optical Kerreffect (MOKE), and superconducting quantum interference device (SQUID) when needed.

\section{B. Pump-probe technique}

To measure the magnetization precession, we use a femtosecond laser in a pump-probe experiment with direct optical excitation, very similar to the method introduced by Ju et $a l^{4}$ to investigate a $\mathrm{Ni}_{81} \mathrm{Fe}_{19} / \mathrm{NiO}$ exchange-biased system. In the measurements, a $150 \mathrm{fs}$ pulse with energy of $40 \mathrm{~nJ}$ excites the sample (pump), and then another weaker pulse is (a)

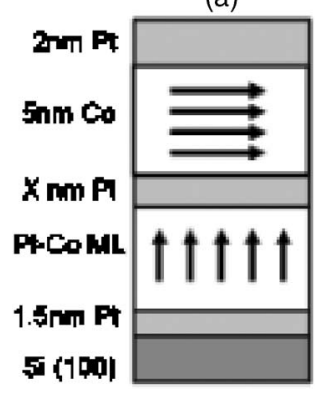

(a)

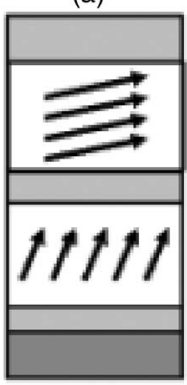

FIG. 1. A schematic of the sample structure and the orientation of the magnetizations in the Co and Pt-Co layers (a) with no coupling between the layers and (b) with ferromagnetic coupling between the layers. 
used to probe the magneto-optical Kerr effect of the sample. This method maps out the direction of magnetization as a function of time, following the initial perturbation from equilibrium. The time delay between the pump and probe pulses is varied by an optical delay line. The probe and pump are focused down to 20 and $35 \mu \mathrm{m}$ spots, respectively. We use a standard double modulation method with an optical chopper and a Faraday modulator to detect pump-induced changes of the Kerr rotation $(\Delta$ Kerr) as a function of the time delay.

The advantages of this technique are the ability to directly measure the dependence of precession frequency on the applied field and to determine the magnetic properties locally only limited by the spot size. It is, however, a thermal excitation which requires the use of thermally conducting substrates. Furthermore, the thermal excitation can simultaneously induce changes in the magnetization, anisotropy, and exchange coupling of the system, placing the system into a highly nonequilibrium state, making it difficult to analyze. An additional challenge, specific to our system, is the simultaneous consideration of two interacting subsystems. If the layers were noninteracting, then the signal would be a superposition of single-layer signals, but the ferro- or antiferromagnetic net interaction between the layers mixes the modes. There are two types of coupled modes, namely ferromagnetic (FM) or "acoustic" modes and antiferromagnetic (AFM) or "optic" modes. The AFM modes are characterized by phase shift of $180^{\circ}$ between the magnetizations of neighboring layers, very similar to traditional antiferromagnetic resonance. ${ }^{7,8}$ However, the present modes refer to different parts of a nanostructure (layers) rather than to atomic sublattices, so that the frequencies are closer to FM resonance than to atomic-scale AFM resonance. ${ }^{9-12}$

\section{Fourier analysis}

To obtain the frequency spectra, the pump-probe data are Fourier transformed. Both $\mathrm{Co}$ and $\mathrm{Pt}$ are metals with a significant spin-orbit interaction, so that the resonance-line analysis includes width and shape of the lines. We use a discrete Fourier transform of our data and perform a least squares fit of the data to a finite Fourier transform of the function,

$$
A_{1} e^{-\beta_{1} t} \cos \left(\omega_{1} t\right)+A_{2} e^{-\beta_{2} t} \cos \left(\omega_{2} t+\varphi\right),
$$

to obtain the frequencies and linewidths. Here, $A_{1,2}$ is the amplitude of each mode, $\beta_{1,2}$ is the effective damping of each mode, $\omega_{1,2}$ is the frequency of each mode, and $\varphi$ is a relative phase between the two modes. To obtain reasonable fits, we must assume that the modes are nearly out of phase with each other. In the following sections we take a phase angle of $180^{\circ}$.

\section{RESULTS AND DISCUSSION}

Both the strength and the angle of an external applied magnetic field were varied, and for many samples, two modes with two distinct precession frequencies were observed. The frequencies of both modes are influenced by the strength and the angle of the applied magnetic field and by the interlayer exchange. Simplifying somewhat, the reso-
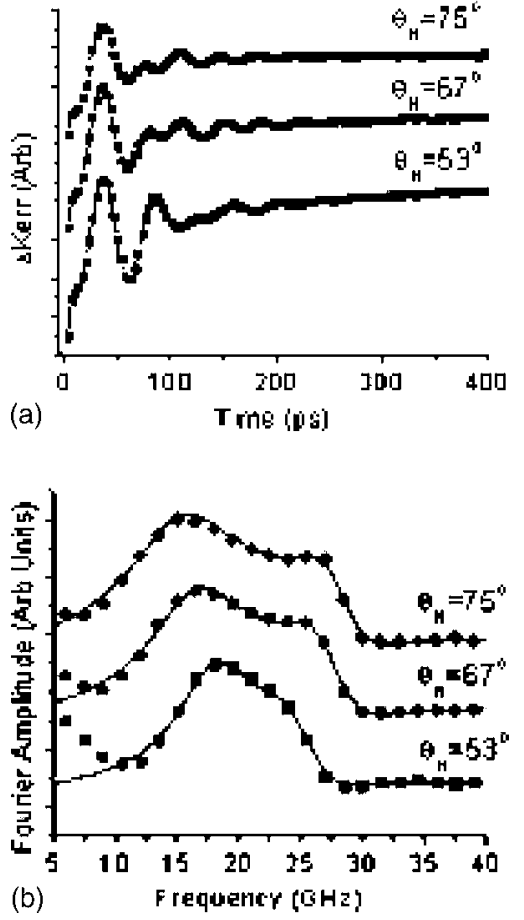

FIG. 2. Typical pump-probe signals as a function of time for the sample with $x=2.1 \mathrm{~nm}$ Pt spacer layer for various angles with respect to the sample normal of a $4.5 \mathrm{kOe}$ applied field. (b) The Fourier transforms of the data in (a) (points) and corresponding least squares fits of the transform (lines).

nance frequency is given by the effective field acting on the spins, and this field depends on field angle and exchange. Figure 2(a) shows the $\Delta$ Kerr signal as a function of delay time for the $x=2.1 \mathrm{~nm}$ sample at various angles of a $4.5 \mathrm{kOe}$ applied field. The angles of the applied field are measured with respect to the sample normal. The clearly visible beats in the precession indicates the presence of two modes. Figure 2(b) shows the discrete Fourier transform of the data (points) and the least squares fit of the finite Fourier transform of Eq. (1) to the data (lines) which gives the mode frequencies and the linewidths. At angles close to $45^{\circ}$ it is difficult to resolve the modes, but at larger angles the mode frequencies separate, clearly showing two distinct frequencies. The frequency-dependent curves were obtained by using the Fourier analysis outlined in Sec. II B. Note that the line shapes in Fig. 2(b) are well reproduced by our Fourier-transformation method, where approximating the lines by a superposition of Gaussian or Lorentzian functions yields much poorer line fittings.

To analyze the data of Fig. 2, we use a model where acoustic and optic modes (Sec. II B) are coupled together by a net interaction, very similar to the approach used by Lindner and Baberschke. ${ }^{11}$ In a linear approximation, ${ }^{12}$ the problem amounts to the diagonalization of a $2 \times 2$ matrix whose diagonal and nondiagonal matrix elements are proportional to the eigenfrequencies of the individual layers and the interlayer coupling $J$, respectively. In this paper, we obtain the two precession modes directly from solving the LandauLifshitz-Gilbert (LLG) equations for two layers of uniform magnetization, $M_{1}$ and $M_{2}$. Here, the first layer has PMA described by second and fourth order anisotropy constants and the second layer has only shape anisotropy. Throughout 


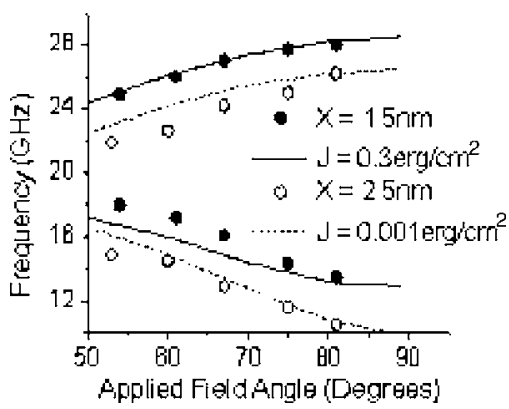

FIG. 3. Mode frequencies as a function of applied field angle for the samples with Pt spacers layers $=1.5 \mathrm{~nm}$ (closed circles) and $2.5 \mathrm{~nm}$ (open circles) and the LLG predicted mode frequencies for coupling constant $J$ $=0.3 \mathrm{erg} / \mathrm{cm}^{2}$ (solid lines) and $J=0.001 \mathrm{erg} / \mathrm{cm}^{2}$ (dotted lines). The magnitude of the applied field was $4.5 \mathrm{kOe}$

each layer, the magnetization is assumed to remain uniform coherent (uniform). This is a reasonable approximation because the leading interactions are vertical, with very little intralayer magnetization variation normal to the film plane.

The two mode makes it possible to estimate the interlayer exchange $J$ from the measured frequency data. The magnetization of an isolated Co film was measured to be $1225 \mathrm{emu} / \mathrm{cm}^{3}$. Measurements on an isolated $\mathrm{Co} / \mathrm{Pt}$ film of the same composition as our coupled films revealed a magnetization of $770 \mathrm{emu} / \mathrm{cm}^{3}$ and an estimated uniaxial anisotropy $K_{1}=4.1 \times 10^{6} \mathrm{ergs} / \mathrm{cm}^{3}$. These values were used as inputs into the LLG model, although the value of $K_{1}$ was varied over about $10 \%$ to improve the resulting fits. As an example, Fig. 3 shows how the mode frequencies depend on the angle of the applied magnetic field for the $x=1.5 \mathrm{~nm}$ (solid symbols) and $2.5 \mathrm{~nm}$ (open symbols) samples. The solid curves are the two mode frequencies predicted by the LLG coupled-mode model for the $x=1.5 \mathrm{~nm}$ sample, with $J=0.3 \mathrm{erg} / \mathrm{cm}^{2}$. The agreement with the experimental results for the higher frequency mode is excellent, but the agreement with the lower frequency mode is only semiquantitative. Similarly, the dashed curves are those predicted by the LLG coupled-mode calculations for the $x=2.5 \mathrm{~nm}$ sample, with $J=0.001 \mathrm{erg} / \mathrm{cm}^{2}$. Here, the model calculations pretty well predict the behavior of the low frequency mode, but agreement for the high frequency mode is less satisfactory. These values of $J$ are consistent with hysteresis loop measurements on our samples and on a related thin-film structure, $J=0.5 \mathrm{erg} / \mathrm{cm}^{2}$ for $\mathrm{Sm}-\mathrm{Co} /[\mathrm{Co} / \mathrm{Pt}]$ thin films. It should be noted that the estimated uncertainty in a single measurement is $0.5-1.0 \mathrm{GHz}$, depending on the sample, the mode, and the applied field angle.

Similar comparisons for samples with $x=1.5$ and $2.1 \mathrm{~nm}$ spacer layers lead to estimates of $J=0.3 \mathrm{erg} / \mathrm{cm}^{2}$, while for the $x=1.8 \mathrm{~nm}$ sample, $J=0.1 \mathrm{erg} / \mathrm{cm}^{2}$. For spacer layer thicknesses larger than $2.1 \mathrm{~nm}$, the interlayer coupling drops with increasing thickness. A striking feature is that the exchange constants are positive, as opposed to the oscillatory behavior expected for RKKY-type interactions. Even in our samples with thinner spacer layers, there is no evidence of antiferromagnetic coupling. The reason is the electronic structure of Pt. As for other exchange-enhanced Pauli paramagnets, Pt is close to ferromagnetism, so that a ferromagnetic perturbation (interface) spin-polarizes the neighboring Pt atoms. The enhanced exchange modifies the wave-vectordependent spin susceptibility ${ }^{13}$ and yields, after Fourier transformation, an exponentially decaying preasymptotic ferromagnetic coupling. For very large $\mathrm{Pt}$ thicknesses, the asymptotic RKKY oscillations take over, but this regime is difficult to reach in typical thin-film structures.

\section{CONCLUSIONS}

In conclusion, we have investigated the behavior of Co-Pt multilayers exchange coupled to a Co layer by a Pt layer. Our pump-probe technique makes it possible to identify and separate two resonance modes, namely, an optical mode and an acoustic mode. The optical mode is very similar to the mode investigated in antiferromagnetic resonance, but due to the nanoscale character of the interaction, the frequency of the mode is rather close to that of the ferromagnetic mode. Due to the preasymptotic ferromagnetism of the interlayer coupling, the net exchange is positive but depends on the thickness of the Pt layer.

\section{ACKNOWLEDGMENTS}

This work is supported by NSF-MRSEC, the W. M. Keck Foundation, and the Nebraska Center for Materials and Nanoscience. The authors are grateful to N. I. Polushkin for discussing various details of this work.

${ }^{1}$ P. Grünberg, R. Schreiber, Y. Pang, M. B. Brodsky, and H. Sowers, Phys. Rev. Lett. 57, 2442 (1986)

${ }^{2}$ M. N. Baibich et al., Phys. Rev. Lett. 61, 2472 (1988).

${ }^{3}$ R. Skomski and J. M. D. Coey, Permanent Magnetism (Institute of Physics, Bristol, 1999).

${ }^{4}$ G. Ju, A. V. Nurmikko, R. F. C. Farrow, R. F. Marks, M. J. Carey, and B. A. Gurney, Phys. Rev. Lett. 82, 3705 (1999).

${ }^{5}$ M. van Kampen, C. Jozsa, J. T. Kohlhepp, P. LeClair, L. Lagae, W. J. M. de Jonge, and B. Koopmans, Phys. Rev. Lett. 88, 227201 (2002).

${ }^{6}$ M. C. Weber, H. Nembach, S. Blomeier, B. Hillebrands, K. Kaltofen, J. Schumann, M. J. Carey, and J. Fassbender, Eur. Phys. J. B 45, 243 (2005). ${ }^{7}$ E. S. Dayhoff, Phys. Rev. 107, 84 (1957).

${ }^{8}$ C. Kittel, Introduction to Solid-State Physics (Wiley, New York, 1986).

${ }^{9}$ M. I. Chipara, R. Skomski, and D. J. Sellmyer, J. Magn. Magn. Mater. 249, 246 (2002)

${ }^{10}$ R. Skomski, J. Phys.: Condens. Matter 15, R841 (2003).

${ }^{11}$ J. Lindner and K. Baberschke, J. Phys.: Condens. Matter 15, R193 (2003).

${ }^{12}$ J. Zhou, R. Skomski, S. Michalski, R. D. Kirby, and D. J. Sellmyer, Mater. Res. Soc. Symp. Proc. 887, 139 (2006).

${ }^{13} \mathrm{P}$. Fulde, Electron Correlations in Molecules and Solids (Springer, Berlin, 1991). 